\title{
Thermal Polymerization of 1,2-Dithiane
}

\author{
Kiyoshi Endo, ${ }^{\dagger}$ Tomotsugu SHIROI, and Naoki Murata \\ Department of Applied Chemistry, Graduate School of Engineering, Osaka City University, \\ Sugimoto, Sumiyoshi-ku, Osaka 558-8585, Japan
}

(Received January 17, 2005; Accepted April 1, 2005; Published July 15, 2005)

\begin{abstract}
Thermal polymerization of six-membered cyclic disulfide, 1,2-dithiane (DT) was investigated under various polymerization conditions. The polymerization of DT depends strongly on the monomer concentration, and the polymerization did not proceed at the monomer concentration below $4.0 \mathrm{~mol} / \mathrm{L}$ in feed. In the polymerization of DT in benzene, the polymerization did not effectively proceed even in the presence of typical radcial initiators. From a kinetic study, overall activation energy for bulk polymerization of DT was estimated to be $22.6 \mathrm{~kJ} / \mathrm{mol}$. The polymerization of DT was inhibited by an addition of radical inhibitors, and decreased remarkably in the presence of thiol compounds, indicating that the propagation proceeds by a radical intermediate. The molecular weight of the polymers increased with reaction time. Namely, the cyclic polymer is formed mainly by back biting reaction mechanism during reaction, which is related to the formation of polycatenane. [DOI 10.1295/polymj.37.512]

KEY WORDS Cyclic Disulfide / Ring-opening Polymerization / 1,2-Dithiane / Kinetic Study / Cyclic Polymer / Radical Species /
\end{abstract}

There are many approaches to prepare polymers containing disulfide bonds in the main chain. ${ }^{1-10} \mathrm{~A}$ ring-opening polymerization of cyclic disulfides is one way for synthesizing such polymers. ${ }^{2-8}$ In ringopening polymerizations of cyclic compounds, a equilibrium monomer and polymer should be considered. ${ }^{11-13}$ In the polymerizations of cyclic disulfides, a torsional strain of $-\mathrm{C}-\mathrm{S}-\mathrm{S}-\mathrm{C}-$ bond angle seems to play a role to determine a activity for the polymerization. . $^{9} 10,14$

Recently, much attention has been paid to synthesis of interlocked polymers such as polyrotaxanes and polycatenanes consisting of cyclic components. Especially, synthesis of polycatenane seems to be more difficult than that of polyrotaxane. Thus, many special approach has been reported. During the study of polymerization and characterization of 1,2-dithiane (DT), six-membered cyclic disulfide, we found that polycatenane can be synthesized by the polymerization of DT. ${ }^{15}$ However, some polymerization mechanisms on the thermal bulk polymerization of DT are still obscure. If the formation mechanism of polycatenane formation is clear in the polymerization of DT, more efficient synthesis of polycatenes will be considered. Thus, we study of the polymerization of DT to clarify the mechanism. In this article, we report on the polymerization of DT under various polymerization conditions.

\section{EXPERIMENTAL}

\section{Reagents}

Benzoyl peroxide (BPO), 2,2'-azobisisobutyronitrile (AIBN) and dimethyl 2,2'-azobisisobutyrate (MAIB) were used after recrystallization for several times. Commercially available benzyl mercaptan (BM) and 1,4-butanedithiol (BDT) (Aldrich Co.) were used without further purification. Other reagents were purified by conventional methods. DT was synthesized according to procedures described in a previous paper. ${ }^{15}$ DT synthesized was purified by recrystallization from methanol several times to remove BDT completely, and the highly purified DT was used as the monomer for polymerization. The structure of monomer was checked by ${ }^{1} \mathrm{H}$ and ${ }^{13} \mathrm{C}$ NMR spectra. Melting point of DT synthesized was $31.0-31.5^{\circ} \mathrm{C}$ (lit. $\left.31-32^{\circ} \mathrm{C}\right) .{ }^{9}, 17$

\section{Polymerization Procedure}

Polymerization was carried out in a sealed glass tube. After required amounts of reagents were charged, the tube was degassed, and then sealed under high vacuum. After the polymerization, the tube opened, and contents of the tube were poured into a large amount of $n$-hexane or methanol to precipitate the polymer formed. The polymers were washed well with $n$-hexane or methanol, and dried under vacuum at room temperature. The polymer yield was determined by gravimetry.

†To whom correspondence should be addressed (TEL\&FAX: +81-6-6605-2697, E-mail: endo@a-chem.eng.osaka-cu.ac.jp). 


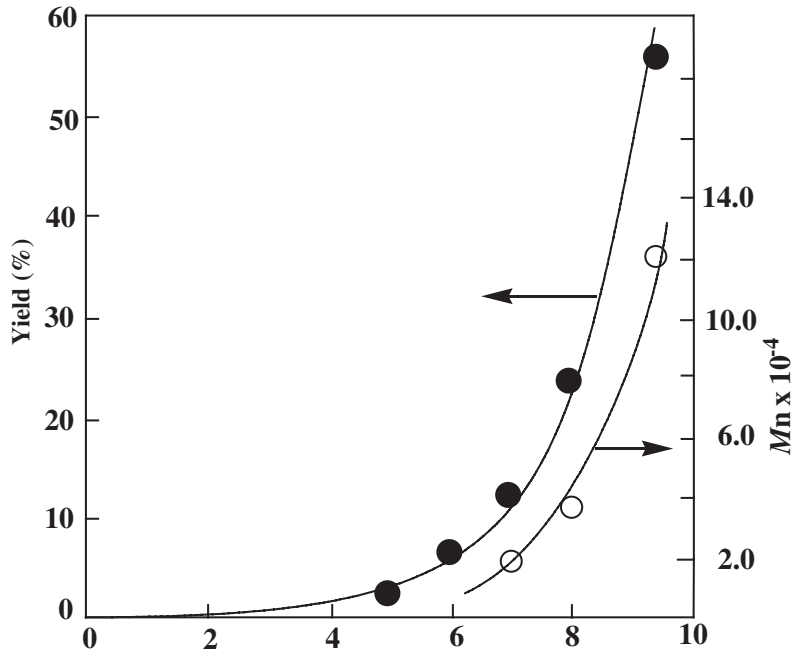

Monomer Concentration (mol/L)

Figure 1. Dependence of monomer concentration for the polymerization of DT in benzene at $60^{\circ} \mathrm{C}$ for $5 \mathrm{~h}$.

\section{Characterization of the Polymers}

The number-average molecular weight $\left(M_{\mathrm{n}}\right)$, the weight-average molecular weight $\left(M_{\mathrm{w}}\right)$, and the molecular weight distribution $\left(M_{\mathrm{w}} / M_{\mathrm{n}}\right)$ of the polymers were determined by GPC using Tosoh CCPD RE-8000 G2000H, G4000H and G6000H columns eluted with THF at flow rate of $1 \mathrm{~mL} / \mathrm{min}$ at $38^{\circ} \mathrm{C}$. Standard polystyrenes were used for calibration of molecular weight of the polymers. The structures of monomer and polymers were analyzed by NMR spectroscopy using a JEOL A-400 spectrometer in $\mathrm{CDCl}_{3}$ with TMS as an internal standard.

\section{RESULTS AND DISCUSSION}

\section{Effect of DT Concentration}

In a previous paper, we reported that bulk polymerization of DT proceeded easily without any radical initiators above the melting point of DT to give a high molecular weight polymer. ${ }^{15}$ Monomer concentration is well known to influence to the polymerization, so that the effect of DT concentration on the polymerization in benzene at $60^{\circ} \mathrm{C}$ was investigated. The results are shown in Figure 1. The polymerization of DT decreased markedly with a decrease of DT concentration in feed, and the polymerization hardly took place at the monomer concentration below $4.0 \mathrm{~mol} / \mathrm{L}$ in feed. In addition to the polymer yields, the molecular weights of polymers also decreased with a decrease of DT concentration. Namely, it is clear that a concentration of the monomer is an important factor for the thermal polymerization of DT. ${ }^{11,12}$

To determine a kinetic order of the polymerization with respect to the monomer concentration, the poly-

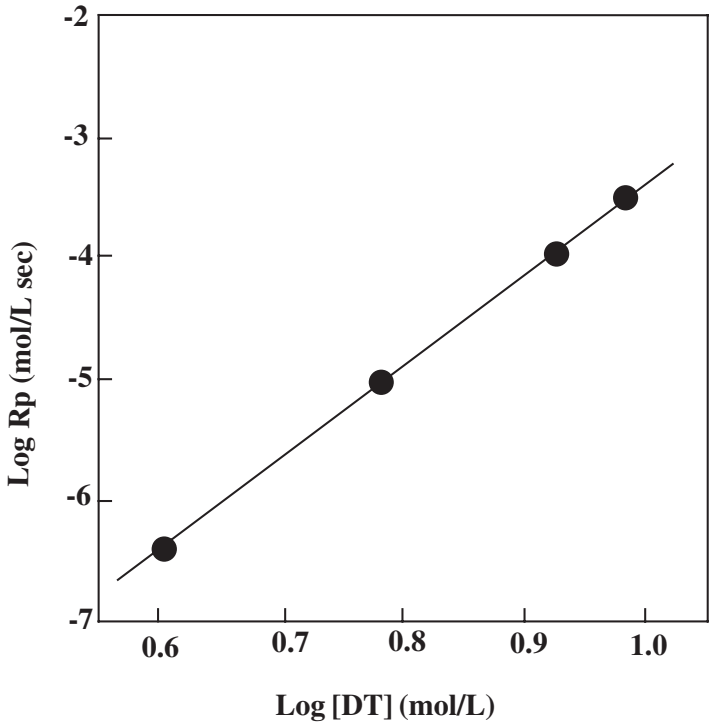

Figure 2. Dependence of DT concentration on $R_{\mathrm{p}}$ for the polymerization of DT in benzene at $60{ }^{\circ} \mathrm{C}$.

merization of DT was carried out at different DT concentrations. The relationships between overall rate of polymerization $\left(R_{\mathrm{p}}\right)$ and $\log$ [DT] gives a linear line as shown in Figure 2. From a slope of the line, $R_{\mathrm{p}}$ was estimated to be in proportion to 6th order of DT concentration. Although the high order of DT concentration on the rate of polymerization seems to be interesting, the accurate reason is not clear.

\section{Polymerization of DT in the Presence or Absence of Radical Initiator}

Solution polymerization of DT was examined in the presence or absence of a radical initiator in benzene at $70^{\circ} \mathrm{C}$. The results are shown in Table I. Polymer did not obtain with AIBN and MAIB. On the other hand, when BPO was used as an initiator, the polymer with relative high molecular weight was obtained in a very low yield. In the ${ }^{1} \mathrm{H}$ NMR spectrum of the resulting polymer, no signals ascribed to fragments of BPO were observed. Since the polymerization was carried out at DT concentration below $4.0 \mathrm{~mol} / \mathrm{L}$, the propagation reaction did not take place even in the presence of radical initiators. This may be explained by equilibrium between monomer and polymer to lied

Table I. Polymerization of DT in benzene at $70^{\circ} \mathrm{C}^{\mathrm{a}}$

\begin{tabular}{lcc}
\hline Initiator & $\begin{array}{c}\text { Time } \\
(\mathrm{h})\end{array}$ & $\begin{array}{c}\text { Polymer Yield } \\
(\%)\end{array}$ \\
\hline AIBN & 10 & 0.0 \\
MAIB & 10 & 0.0 \\
BPO & 30 & $3.0^{\mathrm{b}}$ \\
\hline
\end{tabular}

${ }^{\mathrm{a}}[\mathrm{DT}]=2.8 \mathrm{~mol} / \mathrm{L}, \quad[$ Initiator $]=6.7 \times 10^{-3} \mathrm{~mol} / \mathrm{L}^{\mathrm{b}} M_{\mathrm{n}}=$ $3.1 \times 10^{-3}, M_{\mathrm{w}} / M_{\mathrm{n}}=1.6$ (Determined by GPC). 


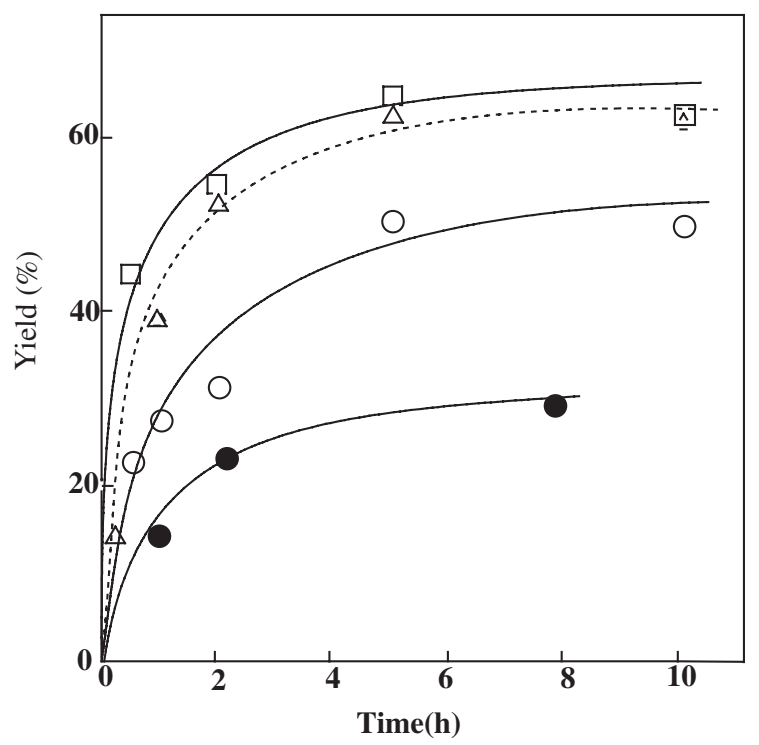

Figure 3. Thermal Polymerization of DT in bulk at $40^{\circ} \mathrm{C}$ $50^{\circ} \mathrm{C}(\bigcirc), 60^{\circ} \mathrm{C}(\triangle)$, and $70^{\circ} \mathrm{C}(\square)$.

to monomer DT for the solution polymerization of DT. ${ }^{11-13}$ However, it is not clear the ceiling temperature and equilibrium monomer concentration of the polymerization of DT.

\section{Effect of Reaction Temperature}

The effect of reaction temperatures on the polymerization of DT was examined. The thermal polymerization of DT was carried out in bulk at 50,60 and $70^{\circ} \mathrm{C}$, respectively. The results are depicted in Figure 3. The rate of the polymerization increased with the temperature. The rate of the polymerization $\left(R_{\mathrm{p}}\right)$ was estimated at the initial stage of the polymerization. The $R_{\mathrm{p}}$ thus determined plotted versus the reciprocal of the reaction temperatures, and the results are shown in Figure 4. From a slope of the Arrhenius plots, the overall activation energy for the polymerization was estimated to be $22.6 \mathrm{~kJ} / \mathrm{mol}$. This may be related to the initiation of the thermal polymerization of DT as will be described below.

\section{Polymerization under Various Conditions}

To clarify the mechanism for the thermal bulk polymerization of DT, the polymerization of DT was investigated under various polymerization conditions. The results are shown in Table II. The polymerization of DT was inhibited almost by the addition of 1,1diphenyl-2-picrylhydrazyl (DPPH) as a radical inhibitor, although a direct observation of thiyl radicals by ESR and an indirect one by a spin trapping method using $N$-tert-butyl-(phenylthio) aminyl oxide were unsuccessful.

The thermal bulk polymerization of DT was suppressed strongly by the addition of thiol compounds

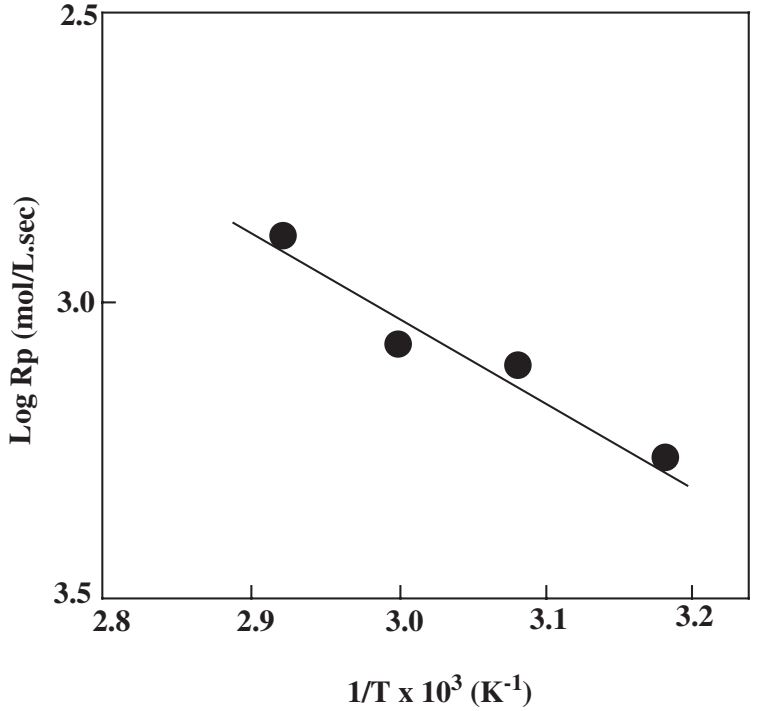

Figure 4. Arrehenius plot for the polymerization of DT in bulk.

Table II. Thermal polymerization of DT in the presence of additives at $80^{\circ} \mathrm{C}^{\mathrm{a}}$

\begin{tabular}{lcrrcc}
\hline Additive & $\begin{array}{c}\text { [Additive }] \\
(\mathrm{mol} / \mathrm{L})\end{array}$ & $\begin{array}{c}\text { Time } \\
(\mathrm{h})\end{array}$ & $\begin{array}{c}\text { Yield } \\
(\%)\end{array}$ & $M_{\mathrm{n}}$ & $M_{\mathrm{w}} / M_{\mathrm{n}}$ \\
\hline- & - & 8 & 83.8 & 28.1 & 2.5 \\
$\mathrm{DPPH}$ & $1.1 \times 10^{-3}$ & 10 & trace & - & - \\
$\mathrm{BM}$ & $8.4 \times 10^{-3}$ & 8 & 3.6 & 10.4 & 2.5 \\
$\mathrm{BDT}$ & $2.4 \times 10^{-3}$ & 30 & 8.6 & 0.9 & 2.5 \\
$\mathrm{H}_{2} \mathrm{O}$ & $1.0 \times 10^{-1}$ & 10 & 46.8 & - & - \\
\hline
\end{tabular}

${ }^{\mathrm{a}}$ In bulk ${ }^{\mathrm{b}}[\mathrm{DT}]=6.0 \mathrm{~mol} / \mathrm{L}$, polymerized in benzene.

such as benzylmercaptane (BM) and butanedithiol (BDT). The $M_{\mathrm{n}}$ of the resulting polymers decreased with an addition of such thiol compounds by taking place a chain transfer reaction. Even when the polymerization of DT was conducted in the presence of a small amount of water, the polymerization of DT was not inhibited. Thus, the propagation of the polymerization of DT seems to proceed via a thyil radical intermediate.

The bond dissociation energies of S-S bond in the monomer structure are usually lower than those of the $\mathrm{C}-\mathrm{C}$ bonds, ${ }^{18-20}$ and the $\mathrm{S}-\mathrm{S}$ bond is like to dissociate by the torsional strain of the cyclic disulfides during the propagation. However, the generation of initiation radical seems to be difficult by the homolysis of the $-\mathrm{S}-\mathrm{S}-$ bond of DT at reaction temperatures examined. ${ }^{20}$ Thus, we presumed that an initiation radical may generate from redox system between DT and a trace of contaminated oxygen in the polymerization tube, although the accurate mechanism is not still clear. We are now investigating the initiation mechanism of the polymerization of DT to clarify this point.

We reported the formation of cyclic polymer from 


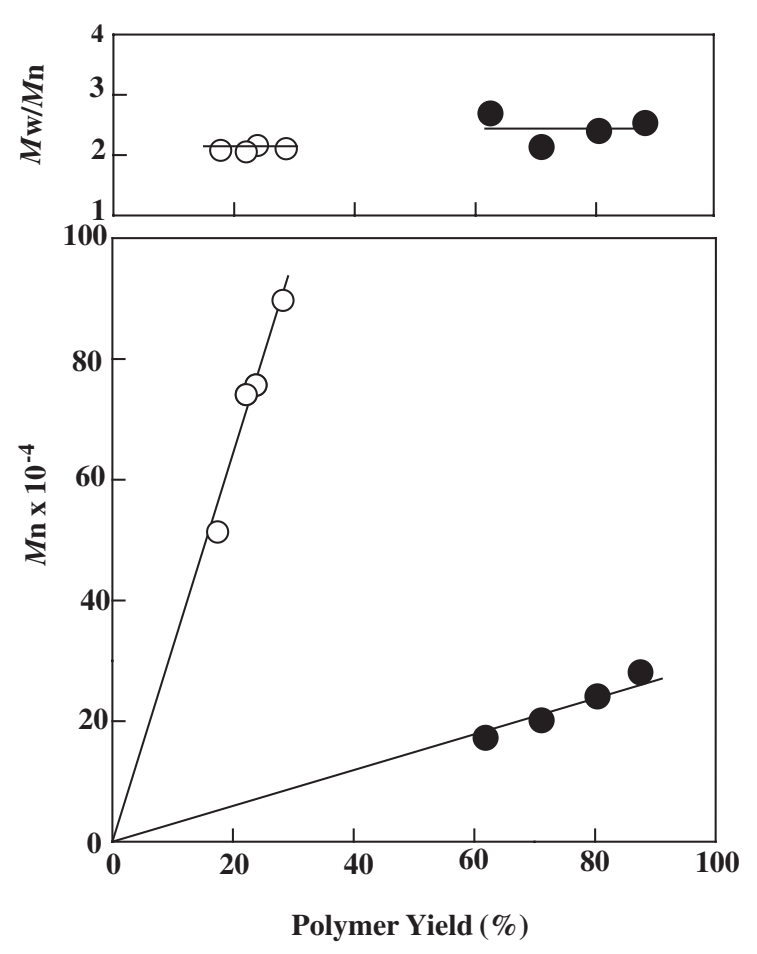

Figure 5. Relationship between the polymer yields and $M_{\mathrm{n}}$ of polymer in the polymerization of DT in bulk at $40^{\circ} \mathrm{C}(\bigcirc)$ and $80^{\circ} \mathrm{C}$

the polymerization of DT in a previous paper. ${ }^{15,16}$ To support this, the polymer yields against molecular weights of the polymer are plotted, and the results are shown in Figure 5. The molecular weight of the polymer increased linearly as a function of a conversion. This can be explained by the formation of interlocked cyclic polymer with a relative low molecular weight throughout reaction, leading to the formation of polycatenane structure. From these results and the structure of the poly(DT), the cyclic polymer was formed by a back biting reaction rather than an intramolecular coupling reaction as reported in a previous paper. ${ }^{15}$ On the basis of the results obtained, the formation of polycatenane was illustrated as shown in Figure 6.

\section{CONCLUSIONS}

Polymerization of DT was carried out under various polymerization conditions. Polymerization of DT strongly depends on the monomer concentration, and no polymer was obtained when the polymerization was carried out at DT concentration below $4.0 \mathrm{~mol} /$ L. In solution polymerization of DT, polymer was hardly obtained at low DT concentration even in the presence of radical initiators. The overall activation energy for the polymerization of DT was low. Thermal bulk polymerization of DT was inhibited by the addition of radical inhibitors such as DPPH. Moreover, polymerization proceeded in the presence of

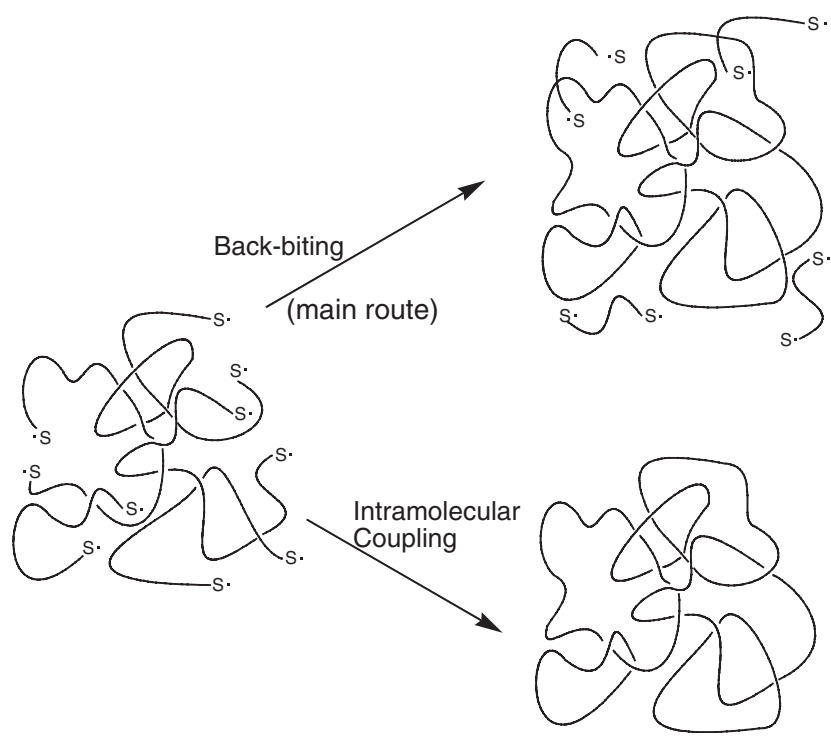

Figure 6. Formation of polycatenane in the polymerization of DT.

water. On the basis of these results, we presume that a trace of oxygen in the system may participate in the initiation mechanism. The relationships between the $M_{\mathrm{n}}$ of the polymer and polymer yields suggest that the cyclic polymer is formed by back biting mechanism in the polymerization of DT.

\section{REFERENCES}

1. R. C. Thomas and L. J. Reed, J. Am. Chem. Soc., 78, 6148 (1956).

2. F. O. Davis and F. M. Fettes, J. Am. Chem. Soc., 70, 2611 (1948).

3. A. V. Tobolsky, F. Leonard, and G. P. Roeser, J. Polym. Sci., 3, 604 (1948).

4. F. S. Dainton, J. A. Davies, P. P. Manning, and S. A. Zahir, Trans. Faraday Soc., 53, 813 (1957).

5. T. Suzuki, Y. Nambu, and T. Endo, Macromolecules, 23, 1579 (1990).

6. J. A. Burns and G. M. Whitesides, J. Am. Chem. Soc., 112, 6296 (1990).

7. R. Singh and G. M. Whitesides, J. Am. Chem. Soc., 112, 6304 (1990).

8. A. Fava, A. Iliceto, and E. Camera, J. Am. Chem. Soc., 79, 833 (1957).

9. J. G. Affleck and G. Dougherty, J. Org. Chem., 15, 865 (1950).

10. J. A. Barltlop, P. M. Hayes, and M. Calvin, J. Am. Chem. Soc., 76, 4348 (1954).

11. S. J. Clarson and J. A. Semylen, Polymer, 27, 91 (1986).

12. R. C. Schulz, K. Albrecht, Q. V. T. Thi, J. Nienberg, and D. Engel, Polym. J., 12, 639 (1980).

13. R. B. Whitney and M. Calvin, J. Chem. Phys., 23, 1750 (1955).

14. J. Houk and G. M. Whitesides, Tetrahedron, 45, 91 (1989).

15. K. Endo, T. Shiroi, N. Murata, G. Kojima, and T. Yamanaka, Macromolecules, 37, 3134 (2004). 
16. R. Arakawa, T. Watanabe, T. Fukuo, and K. Endo, J. Polym. Sci., Part A: Polym. Chem., 38, 4403 (2000).

17. O. G. Lowe, J. Org. Chem., 40, 2096 (1975).

18. S. Oae, in "Organic Chemistry of Sulfur," Plenum Press,
New York, N.Y., 1977.

19. N. S. Isaacs, in "Physical Organic Chemistry," Longman, Essex, U.K., 1987, p 36.

20. S. W. Benson, Chem. Rev., 78, 23 (1978). 